\title{
Absence of a Phase Transition in a Three-Dimensional Vortex Glass Model with Screening
}

\author{
H. S. Bokil and A. P. Young \\ Department of Physics, University of California, Santa Cruz, CA 95064
}

\begin{abstract}
We study the gauge glass model for the vortex glass transition in typeII superconductors, including screening of the interaction between vortices. From the size dependence of the domain wall energy we find that, in twodimensions, the transition is at $T=0$ both with and without screening but the exponents are different in the two cases. In three-dimensions, we find that screening destroys the finite temperature transition found earlier when screening was neglected.

$74.40+\mathrm{k}, 74.60-\mathrm{w}$
\end{abstract}

Typeset using REVTEX 
The question of whether the linear resistance, i.e. the resistance in the limit of a vanishingly small current [1], is really zero in a type-II superconductor in fields greater than $H_{c_{1}}$ has received considerable attention recently, both theoretical and experimental, because fluctuation effects [2] are so much bigger for high $T_{c}$ compounds than for low $T_{c}$ materials. In fact much of the $H-T$ phase diagram of high $T_{c}$ materials is occupied by a "vortex liquid" regime in which the resistance has dropped because superconducting short range order has formed, so flux lines exist locally, but the resistance is not yet zero because the flux lines move under the action of a Lorentz force due to the current, and hence give rise to a voltage [3]. An important question, then, is whether, at lower- $T$, the flux lines can be collectively pinned by defects sufficiently strongly that they have no linear response to the Lorentz force, in which case the linear resistance is zero. Such as state was first proposed for bulk superconductors by M. P. A. Fisher [4] and called the vortex glass. Note that disorder, which is essential to get a vanishing resistance, destroys the flux lattice [5] predicted by mean field theory.

It is important to distinguish whether the dominant type of disorder is point-like, which could be caused by oxygen vacancies in high- $T_{c}$ materials, or whether it is due to extended defects such as twin boundaries or artificially created columnar defects, [6]. The latter case is analogous to bosons moving in a random potential in two dimensions [7]. For this problem the vortex glass state is called a Bose glass [8], which undoubtedly occurs [9] in three dimensions, though some controversies still remain about the details. However, the situation is less clear for point disorder, the case studied here. Much of the theoretical work has studied a simplified model, called the gauge glass. In two dimensions, this has a transition at $T=0$ [10,11, whereas three dimensions seems close to the lower critical dimension [12, 11, though there does appears to be a finite $T_{c}$ [12 in this case. Several experiments [13 on samples of YBCO have found reasonably convincing evidence for a phase transition in non-zero field, presumably to some sort of vortex glass state.

Studies of the vortex glass transition with point disorder have so far neglected screening of the (otherwise logarithmic) interaction between vortices. As we shall see below, screening 
cannot be neglected very close to $T_{c}$. The purpose of this paper is to see if the finite $-T_{c}$ vortex glass transition survives the introduction of screening. We find, at least for the gauge glass model, that it does not. Hence, assuming that the gauge glass model is in the same universality class as experimental systems with point disorder, the observed "transition" in these systems would actually be rounded out sufficiently close to $T_{c}$.

Screening will alter the critical behavior when the penetration depth, $\lambda$, becomes equal to the correlation length, $\xi$, because the important fluctuations on scale $\xi$ will no longer be screened. The behavior of $\lambda$ and $\xi$ are sketched in Figure 1. In the mean field regime, both $\lambda$ and $\xi$ diverge as $t^{-1 / 2}$, where $t=T-T_{c}$, so the ratio $\lambda / \xi \equiv \kappa(>1)$ is roughly constant and is a property of the material. As the temperature is reduced there will be a crossover from mean field behavior to the critical behavior of an uncharged superfluid, in which screening can be neglected, at the Ginzburg temperature, $T_{G}$ [2]. In this critical regime, $\xi$ increases faster than $\lambda$ [2], so the two lengths eventually become equal at $T=T_{s c}$, say. Screening effects will therefore be important for $T_{c}<T<T_{s c}$. Since $\xi\left(T_{s c}\right) \sim \lambda\left(T_{s c}\right) \sim \Lambda_{T}$, we have $\xi\left(T_{s c}\right) / \xi\left(T_{G}\right) \sim \kappa^{2}$, see Figure 1. Now $\kappa \simeq 100$ for high $T_{c}$ compounds so the region where screening is important is small and has probably not been accessed in experiments so far. Nonetheless we feel that it is useful to study the behavior in this region, because (i) this regime may be accessible in experiments on certain materials and ranges of field, and (ii) theoretically, it is interesting to know whether or not the linear resistance is really zero.

Next let us discuss the gauge glass which we model used to study effects of screening. Including a fluctuating vector potential, the Hamiltonian is

$$
\mathcal{H}=-\sum_{\langle I, J\rangle} \cos \left(\phi_{I}-\phi_{J}-A_{I J}-e \mathcal{A}_{I J}\right)+\frac{1}{2} \sum_{\square}(\vec{\nabla} \times \overrightarrow{\mathcal{A}})^{2}
$$

The phase of the condensate, $\phi_{I}$, is defined on each site, $I$, of a regular lattice, square for two dimensions, and simple cubic for $d=3$, with $N=L^{d}$ sites. For now we assume periodic boundary conditions. The sum is over all nearest neighbor pairs on the lattice. The effects of the external magnetic field and disorder are represented by the quenched vector potentials, $A_{I J}$, which we take to be independent random variables with a uniform distribution between 
0 and $2 \pi$. The fluctuating vector potential on each link, $\mathcal{A}_{I J}$, is integrated over from $-\infty$ to $\infty$ [14]. In the last term, which is the usual magnetic energy, the sum is over all elementary squares on the lattice, and the curl, $\vec{\nabla} \times \overrightarrow{\mathcal{A}}$, is the directed sum of the vector potentials round the square, taking into account that $\mathcal{A}_{I J}=-\mathcal{A}_{J I}$. The "charge", $e$, is a measure of the coupling between the condensate and the fluctuating vector potential. The previously studied model [10,12], which neglects screening, corresponds to $e=0$.

It is technically easier to study this model in the vortex representation rather than the above phase representation. To do this we replace the cosine by the periodic Gaussian (Villain) function [15], and perform standard manipulations [15, 16]. For our purposes it is also essential to incorporate the periodic boundary conditions, which leads to an additional term [10,17]. For two dimensions, we find that the vortex Hamiltonian is

$$
\mathcal{H}_{V}=-\frac{1}{2} \sum_{i, j}\left(n_{i}-b_{i}\right) G(i-j)\left(n_{j}-b_{j}\right)+\sum_{\alpha=x, y} V\left(\Phi^{\alpha}-\epsilon^{\alpha \beta} P^{\beta}\right),
$$

where the $\left\{n_{i}\right\}$ run over all integer values, subject to the "charge neutrality" constraint $\sum_{i} n_{i}=0$, and are interpreted as the vortices, $G(i-j)$ is the screened vortex interaction,

$$
\frac{G(i-j)}{(2 \pi)^{2}}=\frac{1}{N} \sum_{\mathbf{k} \neq 0} \frac{1-\exp \left[i \mathbf{k} \cdot\left(\mathbf{r}_{\mathbf{i}}-\mathbf{r}_{\mathbf{j}}\right)\right]}{4-2 \cos k_{x}-2 \cos k_{y}+e^{2}},
$$

and $V(u)$ is the Villain potential given by

$$
\exp \left[\frac{-V(u)}{T}\right]=\sum_{m=-\infty}^{\infty} \exp \left[-\frac{1}{2 T}(u-2 \pi m)^{2}\right] .
$$

$V(u)$ is periodic with period $2 \pi$, and, at $T=0$, is equal to $u^{2} / 2$ for $-\pi<u<\pi$. The vortices sit on the sites, $i$, of the dual lattice which are in the centers of the squares of the original lattice. The $b_{i}$ are given by $(1 / 2 \pi)$ times the directed sum of the quenched vector potentials on the links of the original lattice which surround the site $i$ of the dual lattice. As usual $\epsilon^{x y}=-\epsilon^{y x}=1, \epsilon^{x x}=\epsilon^{y y}=0$. The function $\Phi^{x}$ is defined by

$$
\Phi^{x}=\sum_{x} A_{x}\left(x+\frac{1}{2}, \frac{1}{2}\right),
$$

and similarly for $\Phi^{y}$. Here we indicate explicitly the $x$ and $y$ coordinates of the lattice points: we use integers for the dual lattice, $x=1,2, \ldots, L$, so the coordinates of the original 
lattice are half-integers. The link variable $A_{x}(x+1 / 2,1 / 2)$ connects sites $(x+1 / 2,1 / 2)$ and $(x+3 / 2,1 / 2)$. The function $\mathbf{P}$ is $2 \pi / L$ times the "dipole moment" of the vortex and flux distribution, i.e.

$$
\mathbf{P}=\frac{2 \pi}{L} \sum_{\mathbf{r}} \mathbf{r}[n(\mathbf{r})-b(\mathbf{r})]
$$

The Hamiltonian is independent of the choice of origin for the coordinate system: choosing a new origin alters $\mathbf{\Phi}$ and $\mathbf{P}$ but it is straightforward to show that the arguments of the Villain functions only change by a multiple of $2 \pi$ and so the functions themselves are unchanged. Notice that for $e=0$ the interaction between vortices is logarithmic but that for $e \neq 0$ this logarithmic variation is screened beyond a distance of order $1 / e$.

In $d=3$ the vortices are integer variables which lie on the links of the dual lattice [16]. We find that the vortex Hamiltonian, including periodic boundary conditions, is

$$
\mathcal{H}_{V}=-\frac{1}{2} \sum_{i, j}\left(\mathbf{n}_{i}-\mathbf{b}_{i}\right) \cdot\left(\mathbf{n}_{j}-\mathbf{b}_{j}\right) G(i-j)+\sum_{\alpha=x, y, z} V\left(\Phi^{\alpha}-\frac{1}{2} \epsilon_{\alpha \beta \gamma} C^{\beta \gamma}\right)
$$

where the $\mathbf{n}_{i}$ are subject both to the global constraint $\sum_{i} \mathbf{n}_{i}=0$, analogous to $d=2$, and to the local constraint, $[\nabla \cdot \mathbf{n}]_{i}=0$ for each site [16]. The vortex interaction, $G(i-j)$, is obtained by replacing the RHS of Eq. (3) by its three-dimensional analogue, $C$ is given by

$$
C^{x y}=\frac{2 \pi}{L^{2}} \sum_{\mathbf{r}} \frac{1}{2}\left[x q^{y}(\mathbf{r})-y q^{x}(\mathbf{r})\right]+\frac{2 \pi}{L} \sum_{\mathbf{r}} \frac{1}{2}\left[x q^{y}(\mathbf{r}) \delta_{y, L}-y q^{x}(\mathbf{r}) \delta_{x, L}\right]
$$

where $\mathbf{q}(\mathbf{r})=\mathbf{n}(\mathbf{r})-\mathbf{b}(\mathbf{r})$, with similar expressions for the other components, and $\boldsymbol{\Phi}$ is defined as in Eq. (5). The physical interpretation of $C$ is quite simple. Consider the change in $C$ when 1 is added to all the $\mathbf{n}_{i}$ on a closed loop in the $x y$ plane. Eq. (8) tells us that $C^{x y}$ is then increased by $2 \pi / L^{2}$ times the area of the loop, while $C^{y z}$ and $C^{z x}$ are unchanged. It is easy to see that this is true if the loop does not go along links [18] at which there is a discontinuity in our labeling of the coordinates of the sites, because only the first term in Eq. (8) then contributes, but it is not complicated to show that the result is true for all loops. A change in the boundary conditions, from, say, periodic to anti-periodic in in the $z$-direction, can be incorporated by adding $\pi / L$ to all the $A_{z}$, and so has the effect of 
changing $\Phi^{z}$ by $\pi$. This can be compensated for, in the arguments of the Villain functions, by forming a vortex loop in the $x y$ plane whose area is $L^{2} / 2$, or a combination of loops with the same total area. A derivation of Eqs. (2) and (17) will be given elsewhere [19].

The gauge glass model has random fluxes with no preferred direction, as distinct from the net applied field which occurs in experimental systems. This difference is irrelevant in an expansion in $6-\epsilon$ dimensions, and so may also be irrelevant in $d=3$, though a firm demonstration of this is lacking.

To investigate whether a transition occurs we calculate the defect wall energy [20]. For a given realization of the disorder, we compute the difference in ground state energy, $\Delta E$, between the system with periodic boundary conditions, and with boundary conditions which are anti-periodic in one direction and periodic in the others. To find the ground state, we start from different random initial configurations and quench down to the nearest local energy minimum. We repeat this many times, keeping track of the lowest energy found so far. We monitor the r.m.s. energy difference, $\Delta E_{r m s}$, discussed below, after a logarithmically increasing number of quenches, $1,3,10, \ldots$ and stop when the last two estimates are virtually identical. For a number of cases we ran for much longer than this and verified that the results were unchanged. We also also performed some calculations in the phase representation, using the Villain form for the interaction, and checked that identical results were obtained.

For frustrated systems, such as the model we study here, the average of $\Delta E$ over all samples will be zero, so we look at the root mean square average, $\Delta E_{r m s}=\left[\Delta E^{2}\right]_{a v}^{1 / 2}$, where $[\ldots]_{a v}$ denotes an average over samples. We investigate the size dependence of $\Delta E_{r m s}$ and define an exponent $\theta$ by

$$
\Delta E_{r m s} \sim L^{\theta}
$$

If $\theta<0$ then large domains cost little energy so, at any finite $T$, the system will break up into domains and long range order will be destroyed. By equating the domain wall energy on the scale of the correlation length with $k_{B} T$ one finds that the correlation length varies with $T$ as $T^{-\nu}$ with $\nu=1 /|\theta|$. If, on the other hand, $\theta>0$ then then the rigidity of the 
ordered state should persist to finite temperature.

Our results for $d=2$ are presented in Figure 2 for $2<L<8$. It was not possible to get convergent results for $\Delta E_{r m s}$ for larger sizes. For $e=0$ the slope is $\theta \simeq-0.5$, which is in good agreement with earlier work [10], and gives a correlation length diverging as roughly $1 / T^{2}$. Experimental evidence for this has recently been obtained [21] on very thin films of YBCO. For $e \neq 0$ the slope is clearly more negative, indicating that screening is a relevant perturbation.

The curvature of the data indicates that the intermediate values of $e$ are in a crossover regime. It is therefore be useful to study directly the large $e$ limit. To do this, notice from Eq. (3) that the vortex energy becomes very small in this limit, so the Villain term in Eq. (2) dominates and acts as a constraint fixing the dipole moments of the vortex distribution to the values which minimize the Villain term. Also, in this limit, $G(0)=0$, and $G(i-j)=(2 \pi / e)^{2}$ plus exponentially small corrections which we neglect. Because of charge neutrality, one can subtract the constant $(2 \pi / e)^{2}$ from all the interactions, with the result that only $G(0)\left(=-(2 \pi / e)^{2}\right)$ is non-zero. Hence, for $e \rightarrow \infty, \Delta E_{r m s} e^{2}$ tends to a finite value which can be found by by neglecting the Villain terms in the energy, setting $G(0)=-(2 \pi)^{2}$ and $G(i-j)=0$ for $i \neq j$, and performing a constrained minimization fixing not only the total "charge" but also the total dipole moment of the vortex distribution. On changing the boundary conditions, the $x$-component of the dipole moment is changed by $L / 2$ [22]. Results for $e^{2} \Delta E_{r m s}$ obtained in this way are shown in the inset to Figure 2. The data follows a straight line on a $\log -\log$ plot with a slope of $\theta \simeq-1.36$, which is also roughly the slope of the data for the larger values of $e$ and $L$ in the main part of the Figure.

This result is surprising because there are simple arguments [23] which predict that $\theta=-2$ in the large $e$ limit. One way of seeing this is that, since the vortices don't interact, the probability that a vortex is excited out of its ground state is obtained just by comparing its on-site excitation energy with $k_{B} T$. Since there is a finite density of states, the density of excited vortices is of order $k_{B} T$, and hence a typical separation (which we interpret to be the correlation length) of order $T^{-1 / 2}$, and so $\theta=-1 / \nu=2$. Assuming that this is correct, the 
different result found here must reflect the importance that the constraints (total charge $=0$, fixed dipole moment) have for the rather small system sizes studied here. Note, though, that the presumably correct result, $\theta=-2$, predicts an even faster drop of $\Delta E_{r m s}$ than found in the numerics, and that the numerics is correct in predicting that the screened system is in a different universality class with a weaker divergence at $T=0$.

Our results for $\Delta E_{r m s}$ in $d=3$ are presented in Figure 3. The range of sizes, $L=2,3$ and 4 , is very small, but analogous calculations on similarly small sizes provided one of the first clear indications for the, apparently correct, result that $T_{c}$ is finite in $3-d$ Ising spin glasses 20]. For $e=0, \Delta E_{r m s}$ is roughly independent of size, in agreement with earlier work [12], which indicates that the system is close to the lower critical dimension. For $e \neq 0$, however, $\Delta E_{r m s}$, decreases with increasing size, this effect being more pronounced for larger values of $e$. One can also study the large $e$ limit, as for $d=2$, but we were only able to get convergence for $L=2$ and 3 , and, with only two points, one cannot check for straight line behavior of the log-log plot. Although the sizes are very small, the results of Figure 3 clearly imply that there is no finite temperature transition in $d=3$. The finite temperature transition, for which some evidence was presented in Ref. [12], is therefore rounded out when one includes screening.

To conclude, the presumed finite $T_{c}$ vortex glass transition in the three-dimensional gauge glass model is rounded out by screening effects. The same will be true in real experimental systems with point disorder, assuming that the model is in the same universality class, which is plausible but not yet firmly established. Strictly speaking, the linear resistance would then not vanish, though it would become extremely small because the rounding only occurs very close to $T_{c}$. It would obviously be interesting to investigate this model by finite temperature Monte Carlo simulations, where larger sizes can be studied, and also to simulate a more realistic model with a net field in a particular direction.

We have benefited enormously from stimulating discussions with M. P. A. Fisher. We would also like to thank D. A. Huse for valuable comments. This work is supported by NSF grants DMR 91-11576 and 94-11964. 


\section{REFERENCES}

[1] The voltage is always non-zero in the presence of a finite current because nucleation processes occur, see e.g. [2]. Vanishing of the linear resistance seems to be an appropriate definition of a superconductor.

[2] D. S. Fisher, M. P. A. Fisher and D. A. Huse, Phys. Rev. B. 43, 130 (1991).

[3] See e.g. K. B. Kim and M. J. Stephen, in Superconductivity, edited by R. D. Parks, (Dekker, New York) Vol II.

[4] M. P. A. Fisher, Phys. Rev. Lett. 621415 (1989).

[5] A. I. Larkin and Yu. N. Ovchinikov, J. Low. Temp. Phys. 34, 409 (1979).

[6] D. R. Nelson and V. M. Vinokur, Phys. Rev. B 48, 13060 (1993).

[7] D. R. Nelson, Phys. Rev. Lett. 60, 1973 (1988).

[8] M. P. A. Fisher, P. B. Weichman, G. Grinstein and D. S. Fisher, Phys. Rev. B 40, 546 (1989).

[9] For a recent discussion of the Bose glass transition see M. Wallin, E. S. Sørensen, S. M. Girvin and A. P. Young, Phys. Rev. B 49, 12115 (1994), and references therein.

[10] M. P. A. Fisher, T. A. Tokuyasu and A. P. Young, Phys. Rev. Lett. 66, 2931 (1991).

[11] M. J. P. Gingras, Phys. Rev. B 45, 7547 (1992); M. Cieplak, J. R. Banavar and A. Khurana, J. Phys. A, 24, L145 (1991).

[12] J. D. Reger, T. A. Tokuyasu, A. P. Young and M. P. A. Fisher, Phys. Rev. B 44, 7147 (1991).

[13] R. H. Koch, V. Foglietti, W. J. Gallagher, G. Koren, A. Gupta and M. P. A. Fisher, Phys. Rev. Lett. 63, 1511, (1989); P. L. Gammel, L. F. Schneemener and D. J. Bishop, Phys. Rev. Lett. 66, 953 (991); C. Dekker, W. Eideloth and R. H. Koch. Phys. Rev. 
Lett. 683347 (1992).

[14] Subject to a particular gauge: we choose the gauge in which $\vec{\nabla} \cdot \overrightarrow{\mathcal{A}}=0$. We also set $\overrightarrow{\mathcal{A}}(k=0)=0$. A non-zero $\overrightarrow{\mathcal{A}}(k=0)$ could always compensate for the imposed twist, discussed below, and so we would get the twist energy, $\Delta E$, to be zero. In order to obtain a non-zero result we would then have to apply the twist at a small non-zero wavevector in a transverse direction, see e.g. D. J. Scalapino, S. R. White and S. C. Zhang, Phys. Rev. Lett. 68, 2830 (1992). This is inconvenient for the small sizes studied here.

[15] J. Villain, J. de Phys. (Paris) 36, 581 (1975).

[16] J. V. José, L. P. Kadanoff, S. K. Kirkpatrick and D. R. Nelson, Phys. Rev. B. 16, 1217 (1977); H. Kleinert, Gauge fields in Condensed Matter, (World Scientific, Singapore) (1989).

[17] M. P. A. Fisher, private communication.

[18] Such as the link in the $y$ direction connecting $(x, L, z)$ and $(x, 1, z)$.

[19] H. S. Bokil and A. P. Young (to be published).

[20] A. J. Bray and M. A. Moore, J. Phys. C 17, L463 (1984); W. L. McMillan, Phys. Rev. B 30, 476 (1984).

[21] C. Dekker, P. J. M. Wöltgens, R. H. Koch, B. W. Hussey and A. Gupta, Phys. Rev. Lett. 69, 2717 (1992).

[22] This also works for $L$ odd if one takes, for $L / 2$, the nearest integer. At large but finite $e$, where the Villain function part of $\mathcal{H}_{V}$ is included, there is a strong even-odd effect, because, for $L$ odd, moving the vortices cannot precisely compensate for the change of $\Phi^{x}$ by $\pi$. This is why only data for even $L$ are presented in the main part of Figure 2.

[23] D. A. Huse and M. P. A. Fisher (private communication). 


\section{FIGURES}

FIG. 1. A sketch of the log of the correlation length, $\xi$, and penetration depth, $\lambda$, against $\log t$, where $t=T-T_{c}$, for a type-II superconductor. The slopes of the curves are indicated: $\nu$ is the correlation length exponent in the part of the critical region where screening can be neglected. Two crossovers occur. The first, at the reduced Ginzburg temperature, $t_{G}=T_{G}-T_{c}$ is when critical fluctuations first start to be important. The second, at $t_{s c}=T_{s c}-T_{c}$, is when $\lambda$ and $\xi$ become comparable. Screening effects are important for $t \leq t_{s c}$. For $T>T_{G}, \lambda / \xi=\kappa(>1)$, is constant, while for $T_{s c}<T<T_{G}, \lambda^{2} / \xi$ is constant [2]. Between $T_{G}$ and $T_{s c}$, $\xi$ increases by a factor of $\kappa^{2}$ and $\lambda$ by a factor of $\kappa$.

FIG. 2. A $\log -\log$ plot of the r.m.s. domain wall energy, $\Delta E$, against $L$ for $d=2$ for different values of the coupling $e$. The number of samples varied between 4000 for the smallest sizes to 200 for the largest sizes and values of $e$. For $e=0$ the slope, $\theta$, is approximately -0.5 in agreement with earlier work [10]. For $e \neq 0$ the curves bend down more steeply indicating that screening is a relevant perturbation. The insert shows a $\log -\log$ plot of $e^{2} \Delta E$ against $L$ for $d=2$ in the limit $e \rightarrow \infty$. The number of samples varied between 2000 for $L=2$ and 500 for $L=6$. The line is a least squares fit and has a slope of -1.36 .

FIG. 3. A $\log -\log$ plot of $\Delta E$ against $L$ for $d=3$ for different values of the coupling $e$. The number of samples was 4000 for $L=2$ and at least 1000 for $L=4$. For $e=0$ the slope is close to zero, in agreement with earlier work 12 . For $e \neq 0$ the curves bend down indicating that screening is a relevant perturbation which destroys the (possible) finite temperature transition at $e=0$. 


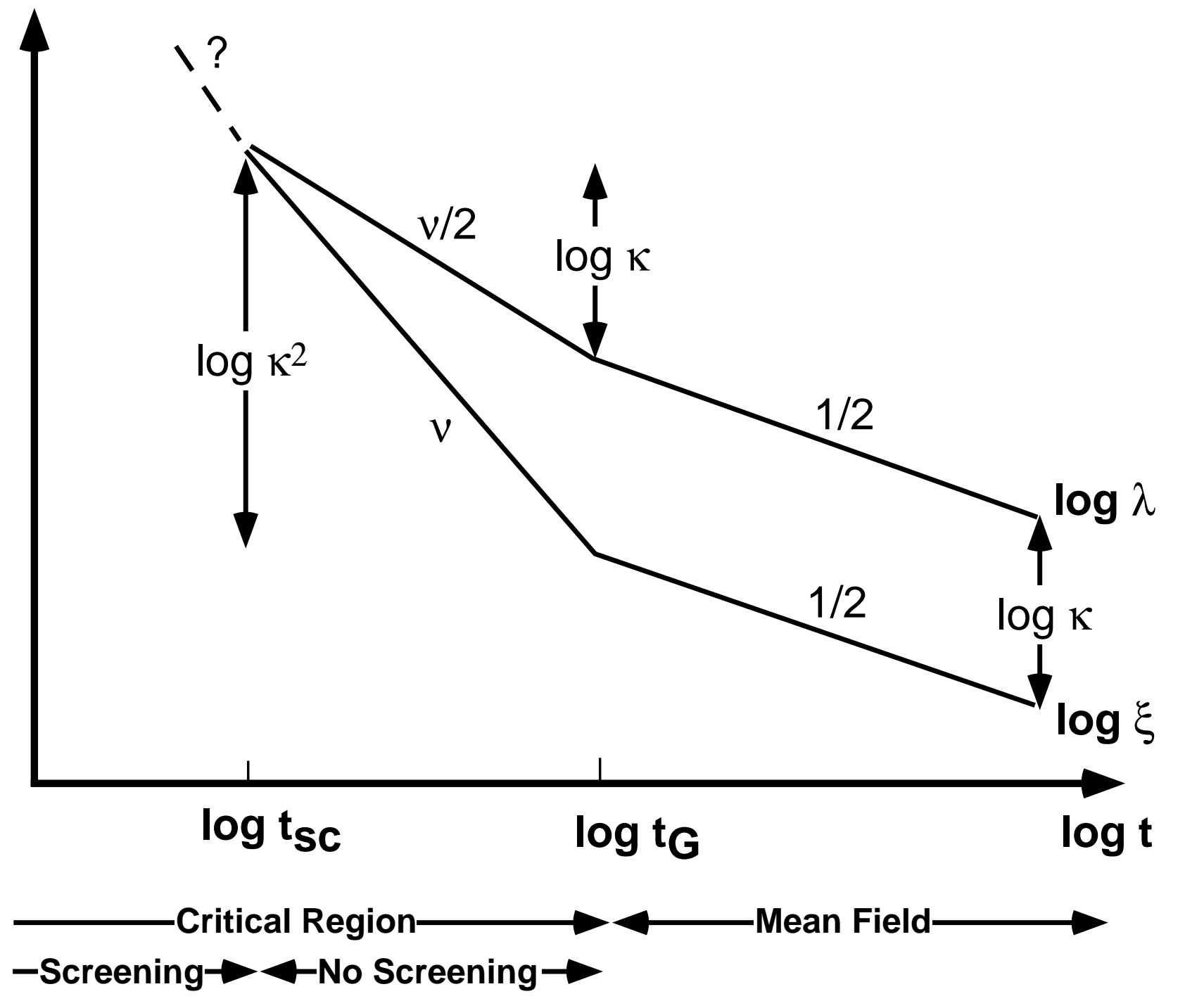

Fig. 1 


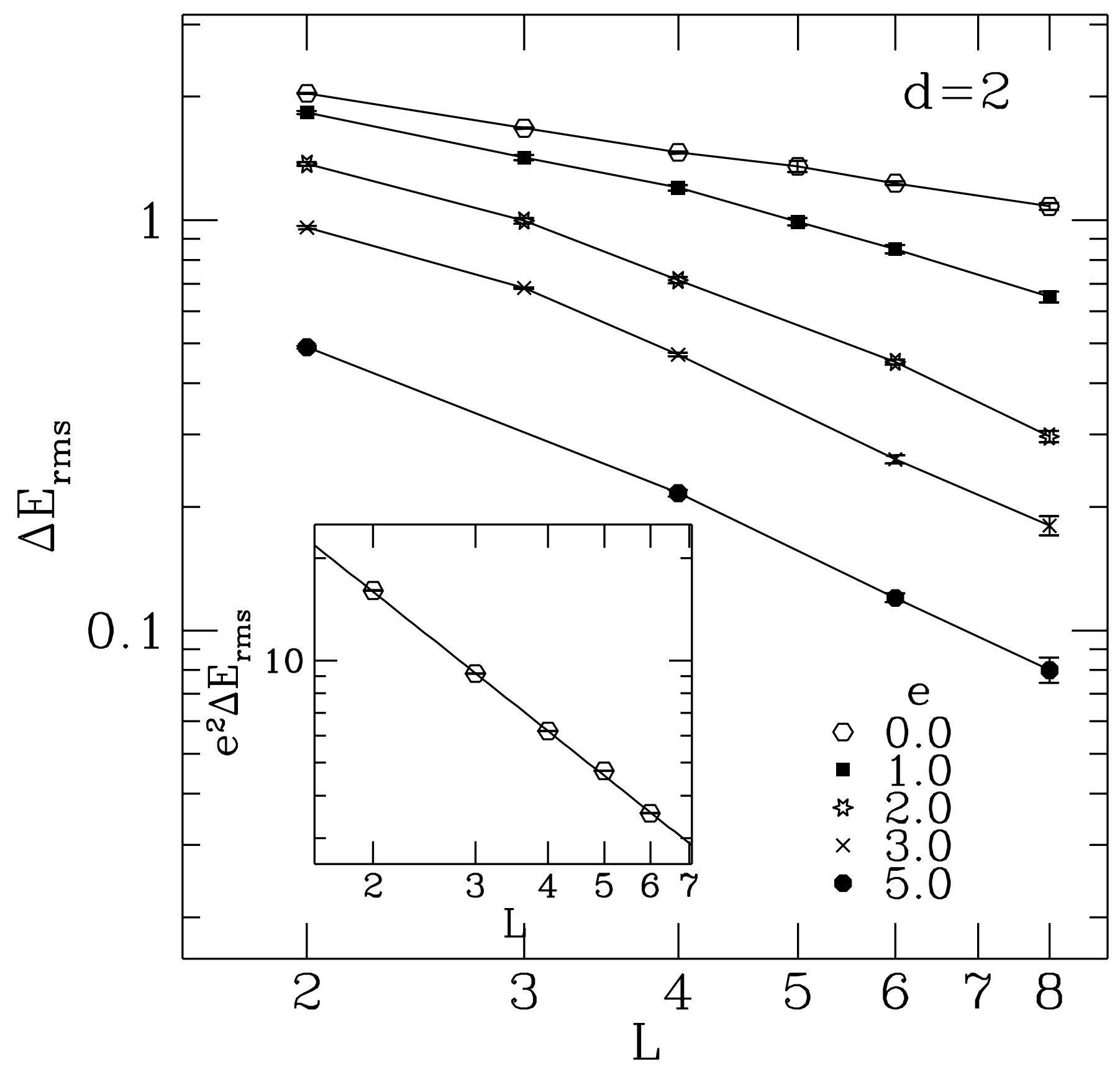

Fig. 2 


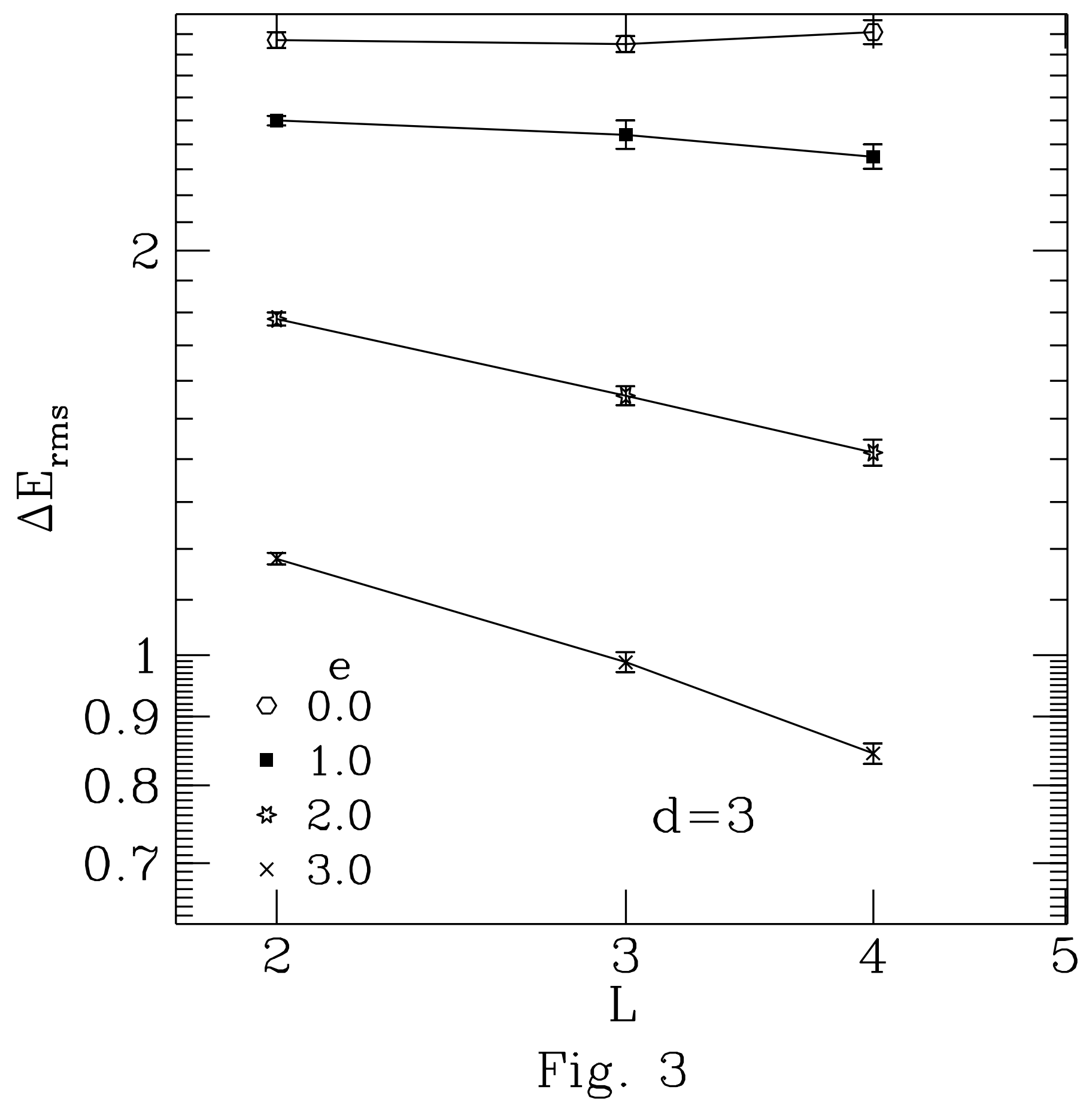

